\title{
CP violation in associated production of a charged Higgs boson and a top quark at the LHC
}

\section{Elena Ginina*}

Institut für Hochenergiephysik der Österreichischen Akademie der Wissenschaften, A-1050

Vienna, Austria

E-mail: eginina@hephy.oeaw.ac.at

\section{Ekaterina Christova ${ }^{1}$, Helmut Eberl ${ }^{2}$}

${ }^{1}$ Institute for Nuclear Research and Nuclear Energy, BAS, Sofia 1784, Bulgaria

${ }^{2}$ Institut für Hochenergiephysik der Österreichischen Akademie der Wissenschaften, A-1050

Vienna, Austria

E-mails: echristo@inrne.bas.bg, helmut@hephy.oeaw.ac.at

\begin{abstract}
We study effects of $\mathrm{CP}$ violation in an associated production of a charged Higgs boson and a top quark at the LHC: $p p \rightarrow t H^{ \pm}+X$. We calculate the CP violating asymmetry between the total cross section for $\mathrm{H}^{+}$and $\mathrm{H}^{-}$production at next-to-leading order in the MSSM, and perform a detailed numerical analysis. In the production only the asymmetry is of the order of $20 \%$. The asymmetry in the production and any subsequent decay of an on-shell charged Higgs boson is to a good approximation the sum of the asymmetry in the production and the asymmetry in the decay. We consider subsequent decays of $H^{ \pm}$to $t b, v_{\tau} \tau^{ \pm}$and $W h^{0}$. In the case of subsequent $H^{ \pm} \rightarrow t b$ decay, the $W^{ \pm}-H^{ \pm}$self energy contributions from the production and the decay cancel. However, the remaining effect, mainly due to $\mathrm{CP}$ violating box graphs with gluino can go up to $\sim 13 \%$.
\end{abstract}

Prospects for Charged Higgs Discovery at Colliders

September 16-192008

Uppsala, Sweden

${ }^{*}$ Speaker. 


\section{Preface}

Recently we studied effects of CP violation (CPV) in the decays of the charged Higgs boson into ordinary particles: $H^{ \pm} \rightarrow t b, H^{ \pm} \rightarrow v \tau^{ \pm}$and $H^{ \pm} \rightarrow W^{ \pm} h^{0}$ in the MSSM [1, 2, 3, 4]. Loop corrections induced by a Lagrangian with complex coupling parameters lead to non zero decay rate asymmetries between the partial decay widths of $H^{+}$and $H^{-}$. We found that in the $H^{ \pm} \rightarrow t b$ decay such effects can be rather large and the asymmetry can go up to $\sim 25 \%$ [1]. This motivated our interest in studying CPV in the production of $H^{ \pm}$at the LHC [5, 6], where the dominant production process is associated with a top quark production: $p p \rightarrow H^{ \pm} t+X$. As we are interested in mass range $m_{H^{+}} \geq 400 \mathrm{GeV}$, at parton level we only consider bottom-gluon fusion: $b g \rightarrow H^{ \pm} t$. The latter process contains the same $H^{ \pm} t b$ vertex and corresponding loop diagrams as the decay $H^{ \pm} \rightarrow t b$, and one would expect that the CPV effects might be of the same magnitude. Moreover, in the production process there are box graphs, that are of the same order and must be taken into account as well, as additional source of CPV.

After the charged Higgs is produced in proton-proton collisions, it will be identified through some of its decay modes. We study CPV in the combined process of $H^{ \pm}$production and decay, considering $H^{ \pm}$decays into $t b, v \tau^{ \pm}$and $W^{ \pm} h^{0}$.

\section{The subprocess $b g \rightarrow t H^{ \pm}$}

We have the following processes, connected by charge conjugation:

$$
\begin{aligned}
& b_{r}\left(p_{b}\right)+g_{\mu}^{\alpha}\left(p_{g}\right) \longrightarrow t_{s}\left(p_{t}\right)+H^{-}\left(p_{H^{-}}\right), \\
& \bar{b}_{r}\left(p_{\bar{b}}\right)+g_{\mu}^{\alpha}\left(p_{g}\right) \longrightarrow \bar{t}_{s}\left(p_{\bar{t}}\right)+H^{+}\left(p_{H^{+}}\right),
\end{aligned}
$$

where $r, s$ and $\alpha$ are colour indices, $r, s=1,2,3 ; \alpha=1, \ldots, 8$.
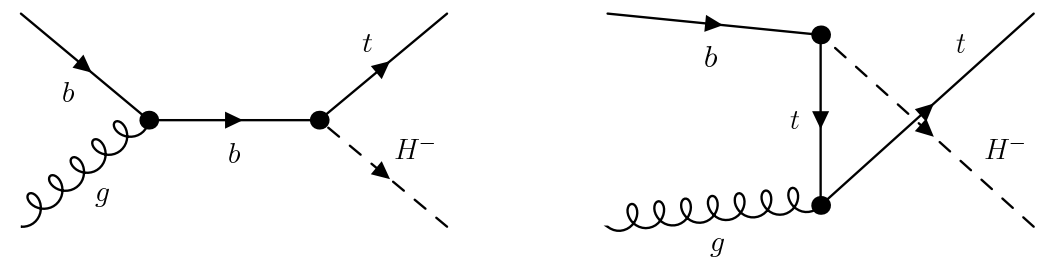

Figure 1: The tree level graphs of the $b g \rightarrow t H^{-}$process.

The tree-level process (2.1) contains two graphs (Fig. 1): with bottom-quark exchange ( $s$ channel), and top-quark exchange ( $t$-channel). The Mandelstam variables are: $\hat{s}=\left(p_{b}+p_{g}\right)^{2}, \hat{t}=$ $\left(p_{t}-p_{g}\right)^{2}=\left(p_{b}-p_{H^{-}}\right)^{2}$.

\section{The LHC process: $p p \rightarrow t H^{ \pm}+X$}

We consider charged Higgs boson production in proton-proton collisions:

$$
p\left(P_{A}\right)+p\left(P_{B}\right) \rightarrow t\left(p_{t}\right)+H^{ \pm}\left(p_{H^{ \pm}}\right)+X .
$$


The Mandelstam variable is: $S=\left(P_{A}+P_{B}\right)^{2}$ ( for LHC $\sqrt{S}=14 \mathrm{TeV}$ ) and we set: $p_{b}=x_{b} P_{A}=\tilde{x}_{b} P_{B}$ and $p_{g}=x_{g} P_{B}=\tilde{x}_{g} P_{A}$, where $x_{i}\left(\tilde{x}_{i}\right)$ is the momentum fraction of the hadron $B(A)$ carried by the parton $i$. Neglecting the proton mass compared to $\sqrt{S}$, we get $\hat{s}=x_{b} x_{g} S=\tilde{x}_{b} \tilde{x}_{g} S$.

We define the CPV asymmetry in the $H^{ \pm}$production as the difference between the total number of produced $H^{+}$and $H^{-}$in proton-proton collisions:

$$
A_{P}^{C P}=\frac{\sigma\left(p p \rightarrow \bar{t} H^{+}\right)-\sigma\left(p p \rightarrow t H^{-}\right)}{\sigma\left(p p \rightarrow \bar{t} H^{+}\right)+\sigma\left(p p \rightarrow t H^{-}\right)}
$$

where the total cross sections in (3.2) are given by:

$$
\sigma^{ \pm}=\sigma\left(p p \rightarrow \bar{t} H^{+}, t H^{-}\right)=2 \int_{0}^{1} f_{b}\left(x_{b}\right) \int_{0}^{1} f_{g}\left(x_{g}\right) \hat{\sigma}^{ \pm}\left(x_{b} x_{g} S\right) \theta\left(x_{b} x_{g} S-S_{0}\right) d x_{b} d x_{g} .
$$

Here $\hat{\sigma}^{ \pm}$are the parton level cross sections for $H^{ \pm}$production in bottom-gluon fusion, $S_{0}=\left(m_{t}+\right.$ $\left.m_{H^{+}}\right)^{2}$ fixes the kinematically allowed energy range, $f_{b}$ and $f_{g}$ are the parton distribution functions (PDF's) of the bottom and the gluon in the proton, $f_{b}\left(x_{b}\right)=f_{\bar{b}}\left(x_{\bar{b}}\right)$, and the factor 2 counts the two possibilities: $b(g)$ comes from the proton $A(B)$ and vice versa.

The CPV asymmetry (3.2) is caused by loop corrections with complex coupling parameters. There are three types of MSSM loop corrections to both $s$ - and $t$-channels that contribute in $A_{P}^{C P}$ : corrections to the $H^{ \pm} t b$-vertex, selfenergy loops on the $H \pm$-line and box diagrams [7]. The total one-loop cross sections of the processes (3.1) have CP invariant and CP violating parts: $\sigma^{ \pm}=$ $\sigma^{i n v} \pm \sigma^{C P}$, and for the asymmetry, up to terms linear in $\alpha_{s}$ and $\alpha_{w}$, we obtain [7]:

$$
A_{P}^{C P}=\frac{\sigma^{C P}}{\sigma^{\text {tree }}} .
$$

\section{4. $H^{ \pm}$production and decay at the $\mathrm{LHC}$}

We define the CPV asymmetry in charged Higgs boson production in $p p \rightarrow t H^{ \pm}$, with a subsequent decay $H^{ \pm} \rightarrow f$, asumming $\mathrm{CPV}$ in both production and decay, as:

$$
A_{f}^{C P}=\frac{\sigma\left(p p \rightarrow \bar{t} H^{+} \rightarrow \bar{t} f\right)-\sigma\left(p p \rightarrow t H^{-} \rightarrow t \bar{f}\right)}{\sigma\left(p p \rightarrow \bar{t} H^{+} \rightarrow \bar{t} f\right)+\sigma\left(p p \rightarrow t H^{-} \rightarrow t \bar{f}\right)},
$$

where $f$ stands for the chosen decay mode: $f=t \bar{b} ; v \tau^{+}$and $W^{+} h^{0}$.

In narrow width approximation, the asymmetry (4.1) is an algebraic sum of the CPV asymmetry $A_{P}^{C P}$ in the production, and the CPV asymmetry $A_{D, f}^{C P}$ in the decay $f$ of the charged Higgs boson $^{1}$ :

$$
A_{f}^{C P}=A_{P}^{C P}+A_{D, f}^{C P}
$$

\section{Numerical analysis}

We present numerical results for the charged Higgs rate asymmetries $A_{P}^{C P}, A_{t b}^{C P}$, and $A_{v \tau}^{C P}$ in the MSSM. All formulas used in the numerical code are calculated analytically and can be found in [7],

\footnotetext{
${ }^{1}$ In $[1,2,3,4]$ and $[8]$ the asymmetry $A_{D}^{C P}$ is denoted with $\delta^{C P}$.
} 
except for the box contributions, which are rather lengthy. Furthermore, all individual one-loop contributions are checked numerically using the packages FeynArts and FormCalc [9]. We also use LoopTools, see again [9], and FF [10]. In the numerical code the Yukawa couplings of the third generation quarks $\left(h_{t}, h_{b}\right)$ are taken to be running [1], at the scale $Q=m_{H^{+}}+m_{t}$. For the evaluation of $f_{b}$ and $f_{g}$ we use CTEQ6L [11], with LO PDF's and NLO $\alpha_{s}$, at the same scale $Q$. We assume GUT relation between $M_{1}$ and $M_{2}$, so that the phase of $M_{1}=0$. Our numerical study showed that the contribution of the loop diagrams with chargino, neutralino, stau and sneutrino in the considered CPV asymmetries are negligible and we show contributions from diagrams with $\tilde{t} \tilde{b}$ and $\tilde{g}$ only. If not specified otherwise, we fix the following MSSM parameters: $M_{2}=300 \mathrm{GeV}, M_{3}=$ $727 \mathrm{GeV}, M_{\tilde{U}}=M_{\tilde{Q}}=M_{\tilde{D}}=350 \mathrm{GeV}, \mu=-700 \mathrm{GeV},\left|A_{t}\right|=\left|A_{b}\right|=700 \mathrm{GeV}, \tan \beta=5, \phi_{A_{t}}=$ $\pi / 2, \phi_{A_{b}}=\phi_{\mu}=0$. The relevant masses of the sparticles for this choice of parameters, $\tan \beta=5$ or 30 are shown in Table 1 of [8]. Our numerical results are in agreements with those in [5], but we disagree analytically and numerically with the results given in [6].

\subsection{Production only}

As expected [1], the CPV asymmetry in the production due to loop corrections with $\tilde{t} \tilde{b}$ and $\tilde{g}$ is of the same order of magnitude as in the case of the decay $H^{ \pm} \rightarrow t b$, and can go up to $\sim 20 \%$. Moreover, the contributions of the box graphs that do not have an analog in the decay is significant and can be dominant for relatively small $m_{H^{+}}$. The contributions of the vertex, selfenergy and box graphs with $\tilde{t} \tilde{b}$ and $\tilde{g}$ to the asymmetry $A_{P}^{C P}$ at hadron level as functions of $m_{H^{+}}$are shown on Fig. 2a. The large effect seen on the figure is mainly due to the phase of $A_{t}$ and the asymmetry reaches its maximum for a maximal phase $\phi_{A_{t}}=\pi / 2$. The phase of $A_{b}$ doesn't have a big influence on the asymmetry and therefore we usually set it to zero.

The asymmetry $A_{P}^{C P}$ reaches its maximum value at $\tan \beta=5$ and falls down quickly with increasing $\tan \beta$. This dependence for $m_{H^{+}}=550 \mathrm{GeV}$ is shown on Fig. $2 \mathrm{~b}$.
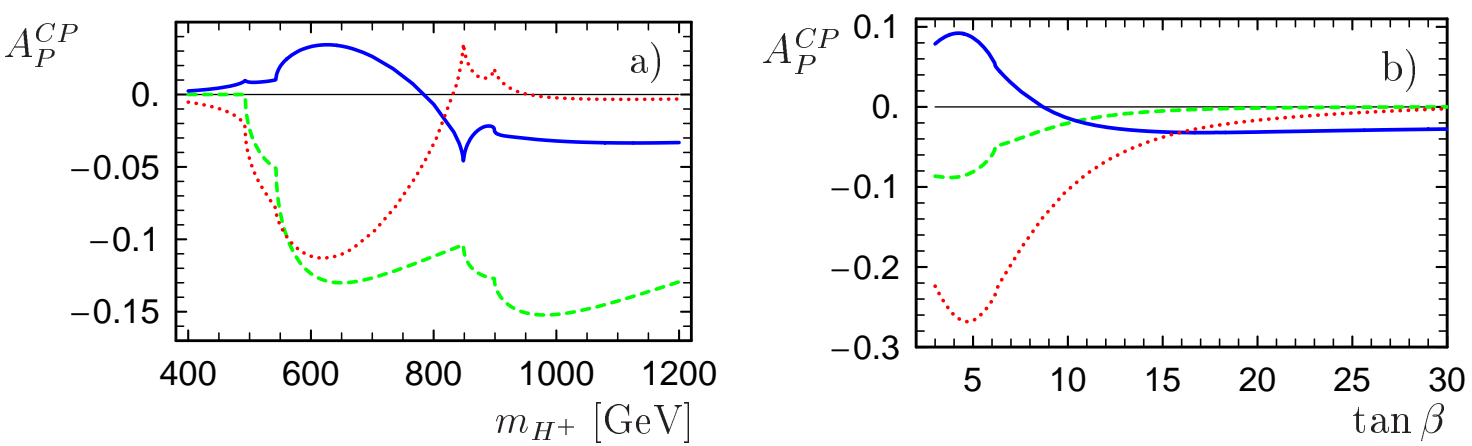

Figure 2: The various contributions to the asymmetry $A_{P}^{C P}$ at hadron level for the chosen set of parameters: a) as a function of $m_{H^{+}}$; b) as a function of $\tan \beta, m_{\tilde{g}}=450 \mathrm{GeV}, m_{H^{+}}=550 \mathrm{GeV}$. The red dotted line corresponds to box graphs with gluino, the solid blue one to the vertex graph with gluino, and the green dashed one to the $W^{ \pm}-H^{ \pm}$selfenergy graph with $\tilde{t} \tilde{b}$ loop.

\subsection{Production and subsequent decay}

First we want to add a few remarks on the branching ratios (BR) of the relevant decays. For small $m_{H^{+}}$, below the $\tilde{t} \tilde{b}$ threshold, the dominant decay mode is $H^{ \pm} \rightarrow t b$, with $\mathrm{BR} \approx 1$, while 
the BR of $H^{ \pm} \rightarrow v \tau^{ \pm}$is in the order of a few percent, decreasing with increasing $m_{H^{+}}$. When the $H^{ \pm} \rightarrow \tilde{t} \tilde{b}$ channels are kinematically allowed, they start to dominate, and the BR of $H^{ \pm} \rightarrow v \tau^{ \pm}$to a good approximation becomes zero. However, the BR of $H^{ \pm} \rightarrow t b$ remains stable of the order of $15-20 \%$, see Fig. 3. The BR of $H^{ \pm} \rightarrow W^{ \pm} h^{0}$ reaches a few percent for small $\tan \beta$ in a relatively narrow range of $m_{H^{+}}$[3]. In the considered range of parameters this decay is very much suppressed and we do not investigate it numerically.

In Fig. 4 a we show the total production and decay asymmetry $A_{f}^{C P}$ at hadron level, for $f=t b$ and $f=v \tau^{ \pm}$. Though for $H^{ \pm} \rightarrow v \tau^{ \pm}$it can go up to $\sim 20 \%$ for $m_{H^{+}} \approx 650 \mathrm{GeV}$, the BR of this decay in this range of $H^{+}$masses is too small and observation at LHC is impossible.

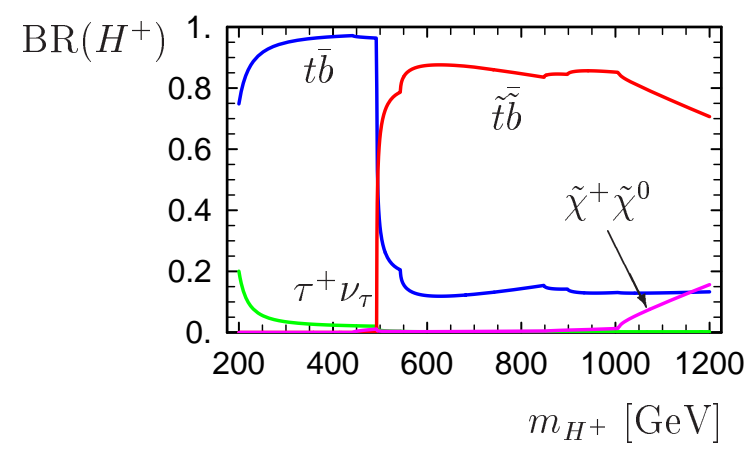

Figure 3: The tree-level branching ratios of $H^{+}$for the chosen set of parameters, as a function of $m_{H^{+}}$.
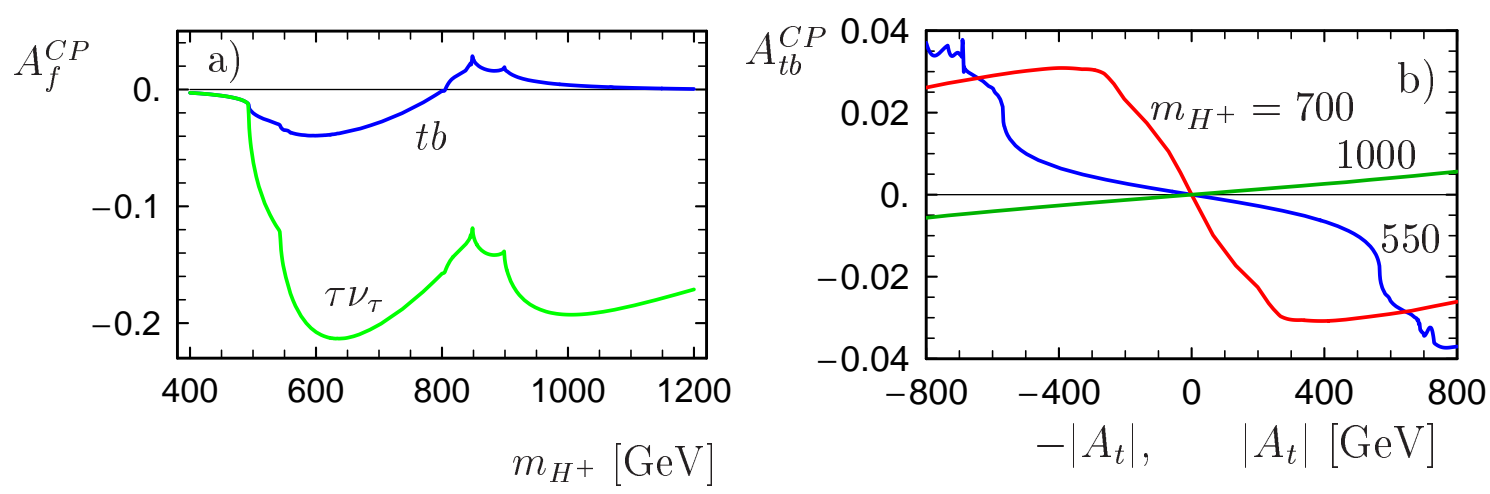

Figure 4: The total asymmetry $A^{C P}$ at hadron level for the chosen set of parameters: a) as a function of $m_{H^{+}}$. The blue line corresponds to the case when $H^{ \pm}$decays to $t b$, and the green one to $H^{ \pm}$decay to $v_{\tau} \tau^{ \pm}$; b) as a function of $\left|A_{t}\right|$, for three values of $m_{H^{+}}$(in GeV).

On the other hand, as the CPV asymmetries in the production and the decay are additive, one can suppose that the total asymmetry will be large. Moreover, in the case of $H^{ \pm} \rightarrow t b$ decay alone it is large [1,8], with leading contribution coming from the $H^{ \pm}-W^{ \pm}$selfenergy graph with $\tilde{t} \tilde{b}$ loop. In [7] we show analytically that the $H^{ \pm}-W^{ \pm}$selfenergy contribution to the asymmetry $A_{t b}^{C P}$ of the decay part cancels exactly the $W^{ \pm}-H^{ \pm}$selfenergy contribution of the production part. Our numerical study showed that the contributions of the vertex graphs from the production and from the decay also partially cancel with the box diagrams contribution. However, as the box graphs do not have a real analogue in the decay, their contribution remains leading in our studied case.

On Fig. $4 \mathrm{~b}$ the dependence of $A_{t b}^{C P}$ on the absolute value of $A_{t}$ is shown for three different $m_{H^{+}}$. 


\section{Summary}

We have calculated the CPV asymmetries $A_{P}^{C P}$, and $A_{f}^{C P}$, with $f=t b ; v \tau^{ \pm}$and $W^{ \pm} h^{0}$, between the total cross sections of $\mathrm{H}^{+}$and $\mathrm{H}^{-}$production in proton-proton collisions, proceeding at parton level through $b g$ fusion. We have performed a detailed numerical analysis, varying the different parameters and phases of the MSSM. The asymmetry $A_{P}^{C P}$ can go up to $\sim 20 \%$ at $m_{H^{+}} \approx 600 \mathrm{GeV}$, $\tan \beta=5$ and a maximal phase of $A_{t}$. This effect is due to CPV vertex, selfenergy and box contributions with $\tilde{t}, \tilde{b}$ and $\tilde{g}$. The total asymmetry in the combined process of production and a subsequent decay is approximately the sum of $A_{P}^{C P}$ and $A_{D, f}^{C P}$, where $f$ is the relevant decay. Despite the dominant CPV contribution from the decay cancels with the relevant part of the production, most promising remains the $t b$ channel. The effect in this case is mainly due to box diagrams with gluino and the asymmetry $A_{t b}^{C P}$ can go up $\sim 13 \%$ [7].

\section{Acknowledgments}

The authors acknowledge support from EU under the MR TN-CT-2006-035505 network programme. This work is supported by the "Fonds zur Förderung der wissenschaftlichen Forschung" of Austria, project No. P18959-N16.

\section{References}

[1] E. Christova, H. Eberl, E. Ginina, W. Majerotto, JHEP 0702 (2007) 075, [hep-ph/0612088].

[2] E. Christova, H. Eberl, S. Kraml and W. Majerotto, JHEP 0212 (2002) 021, [hep-ph/0211063].

[3] E. Christova, E. Ginina, M. Stoilov JHEP 11 (2003) 027, [hep-ph/0307319].

[4] E. Ginina, contribution to the 4th workshop "Gravity, Astrophysics, and Strings at the Black Sea", 2007, [hep-ph/0801.2344].

[5] J. Williams, contributions to CPNSH Report, CERN-2006-009, [hep-ph/0608079].

[6] Kang Young Lee, Dong-Won Jung, H. S. Song, Phys. Rev. D 70 (2004) 117701, [hep-ph/0307246].

[7] E. Christova, H. Eberl, E. Ginina, W. Majerotto, "CP violation in $H^{ \pm} t$ at the $L H C$ ", work in progress.

[8] E. Christova, H. Eberl, E. Ginina, "CP violation in charged Higgs boson decays", contribution to these proceedings, [arXiv:0812.0265 (hep-ph)].

[9] T. Hahn, Nucl. Phys. Proc. Suppl. B 89 (2000) 231; Comp. Phys. Commun. 140 (2001) 418; FeynArts User's Guide, FormCalc User's Guide and LoopTools User's Guide, available at http://www.feynarts.de

[10] G. J. van Oldenborgh, Comput. Phys. Commun. 66 (1991) 1.

[11] J. Pumplin, D.R. Stump, J. Huston, H.L. Lai, Pavel M. Nadolsky, W.K. Tung, JHEP 12 (2002) 0207 [hep-ph/0201195]. 